\title{
5.2. INTERCONTINENTAL LONGITUDE DIFFERENCES OF TRACKING STATIONS AS DETERMINED FROM RADIO-TRACKING DATA*
}

\author{
Donald W. Trask and Charles J. Vegos \\ (Jet Propulsion Laboratory, Pasadena, Calif., U.S.A.)
}

\begin{abstract}
Radio tracking of artificial satellites has provided longitude differences between stations on different continents to $10 \mathrm{~m}$. An accuracy of $1 \mathrm{~m}$ in the future is expected.

\section{RÉSUMÉ}

L'observation par radio des satellites artificiels a fourni des différences de longitude entre stations sur des continents différents à $10 \mathrm{~m}$ près. On espère une précision de $1 \mathrm{~m}$ dans le futur.

\section{Introduction}

The difference between absolute longitudes of two tracking stations located on different continents has already been determined to less than $10 \mathrm{~m}$ while an accuracy on the order of $1 \mathrm{~m}$ is expected in the future.

The determination of these station locations as well as certain physical constants such as the masses of the Earth and Moon, are a by-product of the precise radio tracking of deep space missions performed by the Deep Space Instrumentation Facility (DSIF). The relationship between the knowledge of the spacecraft trajectory obtained from this tracking data and the deep-space station (DSS) location solutions along with the current work and associated limitations are discussed in this paper.
\end{abstract}

\section{Discussion}

The DSIF provides a precise measurement of the change in slant range $(\Delta \rho)$ between the spacecraft and the tracking station over a given time interval $\left(T_{c}\right)$. Currently $\sigma_{\Delta \rho}<10 \mathrm{~cm}$ is obtainable for $T_{c} \leq 10 \mathrm{~min}$ at lunar distances. It has been shown by Hamilton and Melbourne (1966) that the information content of a single pass (horizon to horizon of a single DSS) of Doppler for a distant spacecraft can be compressed

* This paper presents the results of one phase of research carried out at the Jet Propulsion Laboratory, California Institute of Technology, under Contract No. NAS-7-100, sponsored by the National Aeronautics and Space Administration.

Markowitz and Guinot (eds.), Continental Drift, 91-94. () I.A.U. 
into three parameters $(a, b, c)$; namely,

$$
\left.\begin{array}{rl}
a & =\dot{r} \\
b & =\omega r_{s} \cos \delta \\
c & =(\alpha-\lambda-\omega \xi)
\end{array}\right\}
$$

where $\dot{r}=$ probe geocentric radial velocity, $\omega=$ Earth spin rate, $\delta=$ probe declination, $\alpha=$ probe right ascension $\left(\alpha_{\odot} \sim\right.$ instantaneous right ascension of the mean Sun, assumed well known compared to the other parameters), $t_{s}=$ DSS time reference converted to universal time, $\xi=$ error in converting the time reference at the DSS to true universal time, $\Delta \dot{\rho}_{s}=r_{s} \omega \cos \delta \sin \left(\omega t_{s}+\alpha-\alpha_{\odot}-\lambda-\omega \xi\right)=$ range rate due to tracking-station motion, and $\dot{\rho}=\dot{r}+\Delta \dot{\rho}_{s}=$ range rate of probe relative to DSS.

The Doppler tracking data are primarily sensitive to the distance* of the tracking station off the Earth's spin axis $r_{s}$ and the longitude $\lambda$ of the tracking station. Changes in the station location parallel to the Earth's spin axis have little effect on the Doppler data; i.e., the tracking station is essentially located on the surface of a cylinder by the tracking data. Notice that $b$ of equation (1) represents the amplitude of $\Delta \dot{\rho}_{s}$ while $c$ is a measure of the time of meridian passage. It can be seen from equation (1) that stationlocation errors $\left(\varepsilon_{r_{s}}, \varepsilon_{\lambda}\right)$ are highly correlated with probe angular position errors $\left(\varepsilon_{\alpha}, \varepsilon_{\delta}\right)$, and that timing errors directly affect the solution for $\lambda$ by an amount $\omega \xi$. In general, $r_{s}$ and longitude differences are the better determined DSS coordinates. Longitude differences are not affected by timing errors common to the DSS or by errors in $\alpha$. The tracking data directly determines the parameters $a, b$, and $c$; and hence the strength of these determinations is directly related to the quality of the tracking data. However, even if these parameters are perfectly known, the ability to determine tracking-station locations is still dependent upon one's knowledge of the spacecraft coordinates. That is, space missions which result in the probe being significantly influenced by the gravitational field of the Moon (and thus fixing the position of the spacecraft in space), as in the case of the Ranger lunar missions, will produce the best determinations of absolute tracking-station locations.

To date the most intensive postflight analysis has been performed on the four Ranger Block III (Rangers VI-IX) lunar missions. All four flights were tracked continuously from injection to impact on the lunar surface by the use of three DSS; namely, Goldstone, Calif., U.S.A. (DSS 12), Woomera, Australia (DSS 41), and Johannesburg, South Africa (DSS 51). This work was reported in Sjogren et al. (1966) and Vegos and Trask (1967a, 1967b), and current progress is regularly reported in Volume III of the JPL Space Program Summaries, issued bimonthly.

\footnotetext{
* For convenience, this report quotes distances in meters, although the true unit is the light meter (i.e., the distance the electromagnetic signal travels in a unit time is the basic unit of length in the "radio-tracking world"). Throughout the JPL analysis, lengths (in meters) depend on the adopted value of $c=299792.5 \mathrm{~km} / \mathrm{s}$.
} 
During the normal postflight analysis of the tracking data for each Ranger mission, a "best fit" to the tracking data was obtained by adjusting the probe coordinates at an epoch, mass of the Earth, mass of the Moon, and the solar-radiation effect on the spacecraft as well as the tracking-station locations. The longitude differences determined from each flight are compared to a statistical combination of the four flights in Table 1. The maximum deviation from the average for any of the flights is $10 \mathrm{~m}$. The

Table 1

\section{Longitude Differences Referenced to Pole of 1903.0}

Source

Combined Rangers, deg

Ranger VI minus Comb. Rangers

Ranger VII minus Comb. Rangers

Ranger VIII minus Comb. Rangers

Ranger IX minus Comb. Rangers

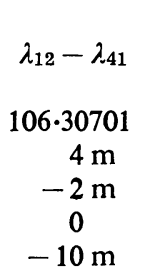

Longitude Differences

$\lambda_{41}-\lambda_{51} \quad \lambda_{51}-\lambda_{12}$

109.20209

$-7 \mathrm{~m}$

$7 \mathrm{~m}$

$-5 \mathrm{~m}$

8

statistical combination was accomplished in a least-squares sense by constraining those parameters common to each flight to a single best value. An exception to this was the Earth-Moon ephemeris scale factor which is allowed to have a unique value for each flight. This is done to compensate for errors which may exist in the lunar ephemeris. The actual statistics of the combined Ranger analysis are probably on the order of $\sigma_{\lambda_{i}-\lambda_{j}} \sim 10 \mathrm{~m}$. In addition to the quality of the tracking data and the uncertainties accounted for is the "ffitters model" of the real universe; this statistic includes uncertainties in the Earth-Moon ephemeris, timing relationships, polar motion, and the effects of the ionosphere. Of these effects, the ionosphere is probably the dominant error source that has yet to be modeled and may account for errors from 5 to $10 \mathrm{~m}$ depending upon the particular flight. It is anticipated that further refinements in the postflight processing of existing tracking data will produce DSS longitude differences approaching $1 \mathrm{~m}$. After the Ranger Block III missions (1965) the DSIF was converted from L-band to S-band which is only $\frac{1}{6}$ as sensitive to the ionosphere. The use of this S-band data may allow these 1-m goals to be exceeded. The navigational accuracy goals being considered for future missions (HAMILTON et al., 1967) will require the knowledge of absolute locations on the order of $1 \mathrm{~m}$.

\section{References}

Hamilton, T.W., Melbourne, W.G. (1966) Information Content of a Single Pass of Doppler Data From a Distant Spacecraft, Space Programs Summary No. 37-39, Vol III, Jet Propulsion Laboratory, Pasadena, Calif.

Hamilton, T. W., Grimes, D. C., Trask, D. W. (1967) Critical Parameters in Determining the 
Navigational Accuracy for a Deep Space Probe During the Planetary Encounter Phase, Space Programs Summary No. 37-44, Vol. III, Jet Propulsion Laboratory, Pasadena, Calif.

Sjogren, W. L., Trask, D.W., Vegos, C.J., Wollenhaupt, W.R. (1966) Physical Constants as Determined From Radio Tracking of the Ranger Lunar Probes, Technical Report 32-1057, Jet Propulsion Laboratory, Pasadena, Calif.

Vegos, C. J., Trask, D. W. (1967a) Tracking Station Locations as Determined by Radio Tracking Data: Comparison of Results Obtained From Combined Ranger Block III Missions and From Baker-Nunn Optical Data, Space Programs Summary No. 37-43, Vol. III, Jet Propulsion Laboratory, Pasadena, Calif.

Vegos, C. J., Trask, D.W. (1967b) Ranger Combined Analysis, Part II: Determination of the Masses of the Earth and Moon From Radio Tracking Data, Space Programs Summary No. 37-44, Vol. III, Jet Propulsion Laboratory, Pasadena, Calif. 